\title{
Evaluation of rotation of Ficus benjamina types in growing environments
}

\author{
Shahzod Egamberdiev ${ }^{1 *}$ and Mansurbek Kholmurotov ${ }^{1}$ \\ ${ }^{1}$ Tashkent State Agrarian University, University str., 2, 100140, Tashkent province, Uzbekistan
}

\begin{abstract}
This article presents the results of research on the evaluation of rooting performance in the growing media of Ficus species in the interior. In the propagation of Ficus species, the vegetative method was selected and experimental work was carried out in growing media of 4 species. Sand (control), soil, Indole Butyric Acid (IBA) $(4,000 \mathrm{mg} / \mathrm{l})$ was obtained as cultivation media. The room temperature at which the experiments were placed was monitored and controlled. During rooting of cuttings, the room temperature was maintained at $18-24^{\circ} \mathrm{C}$. Humidity was maintained at $40-$ $50 \%$. Soil and sand moisture were maintained at 30-40 degrees depending on temperature (based on the readings of the soil moisture meter). Observations were made and recorded every 3-5 days to determine the vegetative rooting rate of the Ficus Benjamin plant. Taking into account the transpiration of the plant and the evaporation of water into the air, 3-5 $\mathrm{ml}$ of water was poured in 10-12 days. As a result of these observations, the timing of vegetative rooting and irrigation norms were determined. Rooted plants were planted in peat, soil, humus feed, coarse sand substrate in a 1:1:1:1 ratio. In selecting these plants, a number of important indicators such as their growth patterns, leaf shape, habitat, drought tolerance, shade tolerance, soil moisture and composition were analysed and conclusions were drawn based on the findings.
\end{abstract}

\section{Introduction}

Modern civilization has given us comfortable homes and comfortable living conditions. But for such benefits, we must first and foremost care about our health. After all, household chemicals, modern construction and decoration materials emit various harmful substances into the atmosphere, constantly polluting our homes and other buildings [1, 2]. Prolonged stay in a polluted room has a negative impact on a person's health and performance, leading to fatigue and various chronic illnesses. Houseplants can best help us with this. Therefore, it is necessary to know how to breed and care for them [2].

As a result of scientific research in the world experience, a number of scientific studies have been conducted on the reproduction of indoor plants. In 2001-2002 and 2002-2003, in such studies on the physiology of Ficus benjamina plants, the rate of plant rooting and transplantation was assessed. It is said that the cutting times of plant cuttings are directly related to the movement of the substances contained in them [3, 4]. As a result, the root rate

* Corresponding author: sh.egamberdiyev@tdau.uz 
of cuttings obtained in February decreased by 34\%, the root rate of cuttings obtained in June decreased by $87 \%$ and in October by $27 \%[1,5]$. Indole Butyric Acid IBA has been shown to reduce plant rooting time. Effective results were obtained when using peat + perlite $(1: 1)$ peat + wood chips $(1: 1)$ as the medium [5].

Several scientists evaluated the growth rates of Ficus benjamina L. plant in different soil environments in their experiments. The effect of peat, perlite, composted tree bark, composted tea waste and rice husk selected as medium on plant height, stem diameter, number of side buds, root development was assessed $[2,6]$. According to the experiments by Babaie and others, they tested plant root performance in 4 types $(0,2,000,4,000$, and $6,000 \mathrm{mg} / \mathrm{l}$ ) of IBA. June was selected for the experiment, and as a result, options 3 and 4 were recommended [3-7].

Khomami and Zadeh studied the effects of processed manure on plant growth. Organic manure $(10 \%, 20 \%, 30 \%$, and $40 \%)$ was used instead of peat as the soil medium $[8,11]$. The compounds in the plant composition that provide the growth rates that affect plant growth are calculated by Mbosso and others [9, 10, 12].

Siddiqui and Hussain conducted their experiments in 2002 on the effectiveness of IBA in propagating ficus species and increasing rooting rates. 1,000, 2,000, 3,000, 4,000, 5,000 ppm and 0 ppm amount options were used as the control option. The 4,000 ppm option was distinguished by its high performance [10,13].

Houseplants with woody stems have been observed to grow more slowly than other categories of species. Such researchers studied the factors influencing the growth of ficus species and the physiology of the plant. This was compared with some similar species [1-4, 14].

Experiments on the propagation of ficus species do not fully allow the plant to grow cheaply and in large quantities on an industrial scale. Therefore, it is important to create cheap, high-quality and effective methods for the reproduction of ficus species. The rooting performance of a plant depends on the timing of its cuttings and the quality of the cuttings. When propagating houseplants, it is necessary to determine at what times of the year cuttings can be obtained [1-7]. Solving problematic situations, such as how long and under what conditions the cuttings are stored, is one of the tasks before us.

When propagating a plant, it is necessary to know its species-specific bioecological features. The effectiveness of the generative or vegetative method in plant propagation should be evaluated. Most houseplants are propagated vegetatively. Our experiments have shown that the method of vegetative cuttings in ficus species is the most effective. As a result of our experiments, the timing of vegetative rooting and irrigation standards are determined.

\section{Materials and methods}

The bioecological characteristics of the plants selected for the experiment were Benjamin ficus (Ficus benjamina) and elastic ficus (Ficus elastica). The experiment was carried out by placing the experimental cuttings in soil, IBA stimulated water, water and sandy environment in the laboratory room and greenhouse. Sand was taken as a control option and the results were compared with the control option. During the experiments, rooting parameters and root rate and its strength were determined in the concentration options. The process of new leaf formation in rooted cuttings was evaluated. The room temperature in which the experiment was performed was monitored and controlled. During rooting cuttings, the temperature is required to be $18-24{ }^{\circ} \mathrm{C}$ and humidity should be $40-50 \%$. Soil and sand moisture was maintained at 30-40 degrees depending on temperature (based on the readings of the soil moisture meter). Observations were made and recorded every 3-5 days to determine the vegetative rooting rate of the ficus benjamin plant. Taking into 
account the transpiration of the plant and the evaporation of water into the air, 3-5 $\mathrm{ml}$ of water was poured in 10-12 days. As a result of these observations, the timing of vegetative rooting and irrigation norms were determined.

Rooted plants were planted in peat, soil, humus feed, and coarse sand substrate in a 1:1:1:1 ratio. Additives made from vermiculite and coniferous bark was added to the substrate to retain moisture. Given that the propagation of houseplants is not the same in different environments; the experiments were carried out in variants in several environments. Depending on the evaporation rate in the liquid medium, additional water was added depending on the moisture level in the soil and sandy environment. Rooting performance was assessed on three criteria and on a 5-point scale.

\section{Results and discussion}

As a result of observations, Benjamin ficus (Ficus benjamina) and elastic ficus (Ficus elastica) were selected for reproduction. Ficus species belong to the family Moraceae, which is native to tropical countries in Africa, Asia and the Americas. The height of the ornamental ficus is 1-1.5 meters, the ficus also blooms, but this phenomenon is very rare. The leaves are 5-10 cm long, 2-5 cm wide, oval, glossy.

Sometimes it drops all its leaves when its location is changed. The plant loves permanence, can tolerate dry air. Ficuses with dark leaves do not require much light compared to light-colored leaves. The plant can tolerate a drop in temperature to $+12{ }^{0} \mathrm{C}$, but it is better not to leave in cold weather. Ficus can burn from direct sunlight, so it should not be left outdoors, even in places where sunlight falls vertically. Washing the leaves of the ficus in the summer gives good results and the leaves grow well. If the humidity in the room decreases sharply, the watering should be increased. Fertilize the plant with universal fertilizer to feed in the fall.

Ficus grows well from rod cuttings. Ampicle-shaped species of ficus take better root than its tree-like and shrub-like forms. After 2-3 weeks in a normal watering can appear lateral roots on the branches placed on it. The leaf blade can be well rooted in spring and summer, for which light is required (except for sunlight). Half-timbered cuttings also take root in moist sand. The cuttings are cut to a length of $8-10 \mathrm{~cm}$ and dipped into water, stimulating water or sand. Propagation at room temperature or in a greenhouse environment gives good results. Rooted cuttings are transplanted into the soil. Ficus pests suffer severely from aphids, thrips. It is treated against diseases and pests using a cold shower or chemical means.

For the interior, ornamental plants are propagated mainly vegetatively. Propagation of houseplants is carried out mainly in room conditions and in special greenhouses. Heat and humidity are important for most plants. To propagate the plants in a liquid medium, a special container is prepared and water or stimulant water is poured into it. For propagation in sand and soil and other similar compounds were used wooden containers or special plastic containers with a height of $15-20 \mathrm{~cm}$, size $0.5 \times 1.0,1 \times 1,1 \times 2$ meters. The tops of the jars were covered with cling film. The fact that most of the houseplants do not bear fruit and seeds, and the seeds are not fully ripe, prevents them from propagating in a generative way. The ficus benjamin plant obtained for propagation was placed in 4 different variants for propagation at the vegetative end and sand was obtained as a control variant (Fig. 1).

As can be seen in Fig. 1, the rooting rates and the rooting rate and its robustness were determined in the variants. The process of new leaf formation in cuttings was evaluated. Room temperature was monitored and controlled. It was noted that observations were made every 3-5 days to determine the vegetative rooting of ficus benjamin plant. Taking into account transpiration and evaporation of water into the air, 3-5 $\mathrm{ml}$ of water was poured in 
10-12 days. As a result of these observations, the timing of vegetative rooting and irrigation norms were determined.

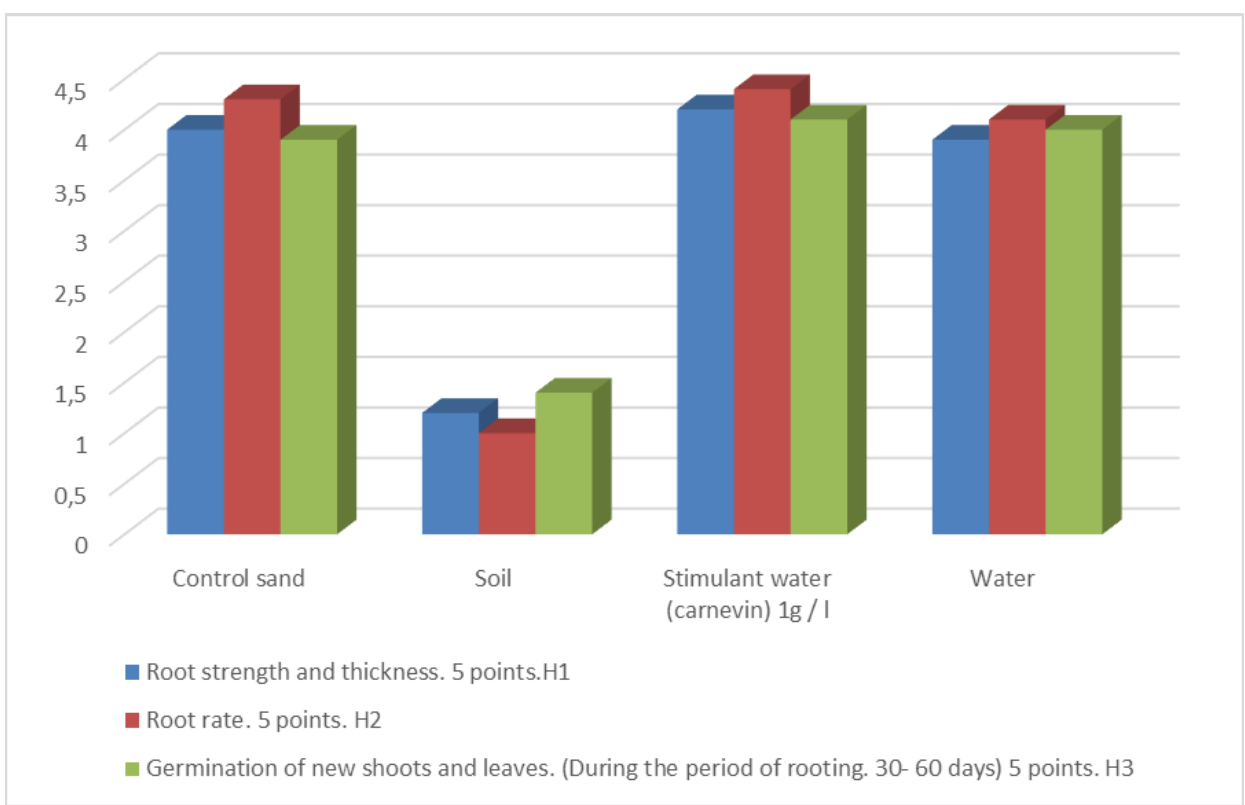

Fig. 1. Experimental results of vegetative propagation of ficus benjamina (Laboratory room of the Department of Ornamental Horticulture, relative humidity of the room is $40-50 \%$ and room temperature is $18-22^{\circ} \mathrm{C}$ ).

In the diagram, root strength and thickness $\left(\mathrm{N}_{1}\right)$, rooting rate $\left(\mathrm{N}_{2}\right)$, new bud and leaf emergence (during rooting period. 30-60 days, $\mathrm{N}_{3}$ ) were evaluated on a 5-point scale.

The experimental options were compared to the control option and the most effective methods were selected. In addition to rooting indicators and markings, external signs of the plant are observed in the control. Experimental options were rated on a 5-point scale. In this case, the number of cuttings in the experiments in each variant was calculated by calculating the number of rooted cuttings, the rate of rooting (Fig. 2).

Calculation of root strength and thickness ( 5 points, $\left.\mathrm{N}_{1}\right)$;

$$
\mathrm{N} 1=\mathrm{V} \times \mathrm{Nm} / \mathrm{A}
$$

where,

A - Number of cuttings (100 pieces).

$\mathrm{V}$ - The number of rooted cuttings ( 80 control options).

$\mathrm{Nm}$ - maximum value 5 points $(100 \%)$

N1 - Root strength and thickness - (0-5)

$$
\mathrm{N} 1=80 \times 5.0 / 100=4.0=\mathrm{N} 1=4.0
$$

Calculation of root rate ( 5 points, $\mathrm{N}_{2}$ );

where,

$$
\mathrm{N} 2=\mathrm{Vo} \times \mathrm{Nm} / \mathrm{A}
$$

A - average (root rate 40 days) 
Vo - Rooting rate $=34$ days (according to the options (30-60).

$\mathrm{Nm}$ - maximum value 5 points

N2 - Rooting rate - (0-5)

$\mathrm{N} 2=34 \times 5.0 / 40=4.25=\mathrm{N} 2=4.25$

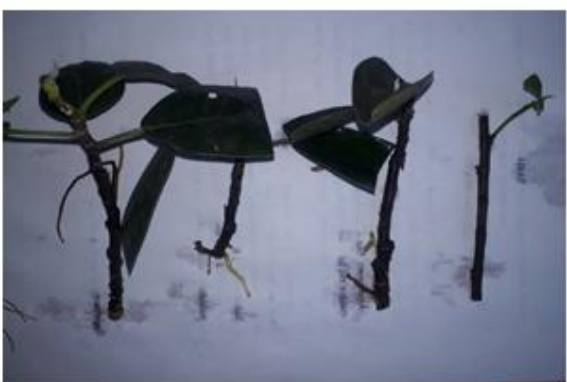

a

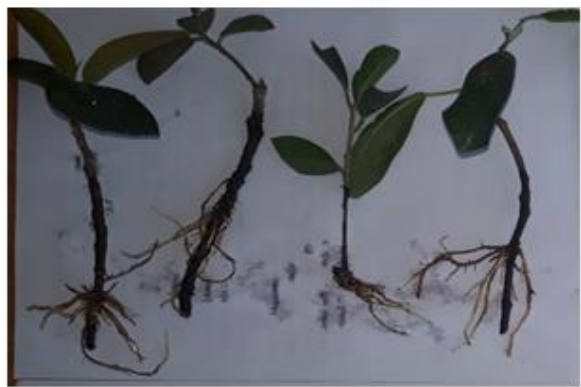

c

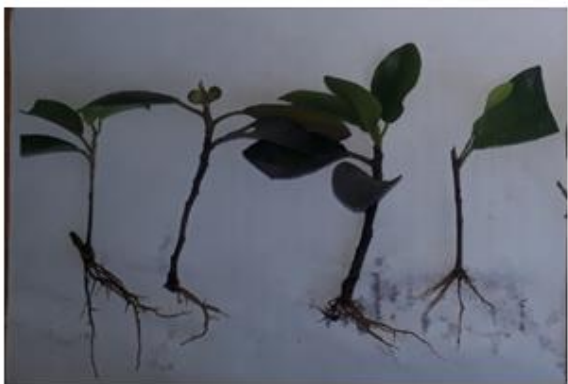

b

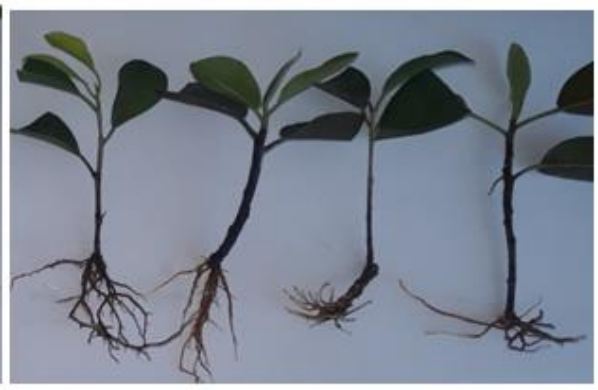

d

Fig. 2. Results obtained in experimental options: a) in the soil, b) in the sand, c) in ordinary water, and d) in carnevin stimulating water.

Calculation of new shoots and leaf emergence ( 5 points, $\mathrm{N}_{3}$ );

$$
\mathrm{N} 3=\mathrm{V} \times \mathrm{Nm} / \mathrm{A}
$$

A - Number of cuttings (100 pieces)

$\mathrm{V}$ - Number of shoots and buds (during the period of rooting. 30-60 days) (78 control options)

$\mathrm{Nm}$ - maximum value 5 points $(100 \%)$

N3 - New bud and leaf emergence - (0-5)

$$
\mathrm{N}_{3}=78 \times 5.0 / 100=3.9=\mathrm{N}_{3}=3.9
$$

The microclimate of the room in the propagation of houseplants, in particular; regulation of air temperature, humidity, light supply gives good results. During rooting of cuttings, the temperature should be $18-24{ }^{\circ} \mathrm{C}$. Humidity should be $40-50 \%$. Soil and sand moisture is maintained at 30-40 degrees (depending on the readings of the soil moisture meter) depending on the temperature. During the vegetative propagation of ficus is demanding to the above indicators. Due to this, the system of applying control measures to them for 3-5 days was considered a tool that had a sufficient impact on their growth and development. 
Houseplants can be propagated using a hanging tie. In this method, several plants are propagated from a growing plant without being removed from the pot. Most houseplants can be propagated using a hanging tie. The disadvantage of the other method is that the plant habit changes. Selection of mainly high and excess branches of the plant In this case, the selected branch of the elastic ficus was removed from the bark up to half of the circumference of the $1 \mathrm{~cm}$ section. A peat bag was tied to the incision site and irrigated every 2-3 days using a syringe. After 25-30 days, the plant was cut from under the bag and the bag was removed and planted in a pot.

Indoor plants can be propagated using a hanging tie. In this case, the selected horn of the elastic ficus was removed from the bark up to half of the circumference of $1 \mathrm{~cm}$. A peat bag was tied to the incision site and irrigated every 2-3 days using a syringe. After 25-30 days, the plant was cut from under the bag and the bag was removed and planted in a pot (Fig. 3).
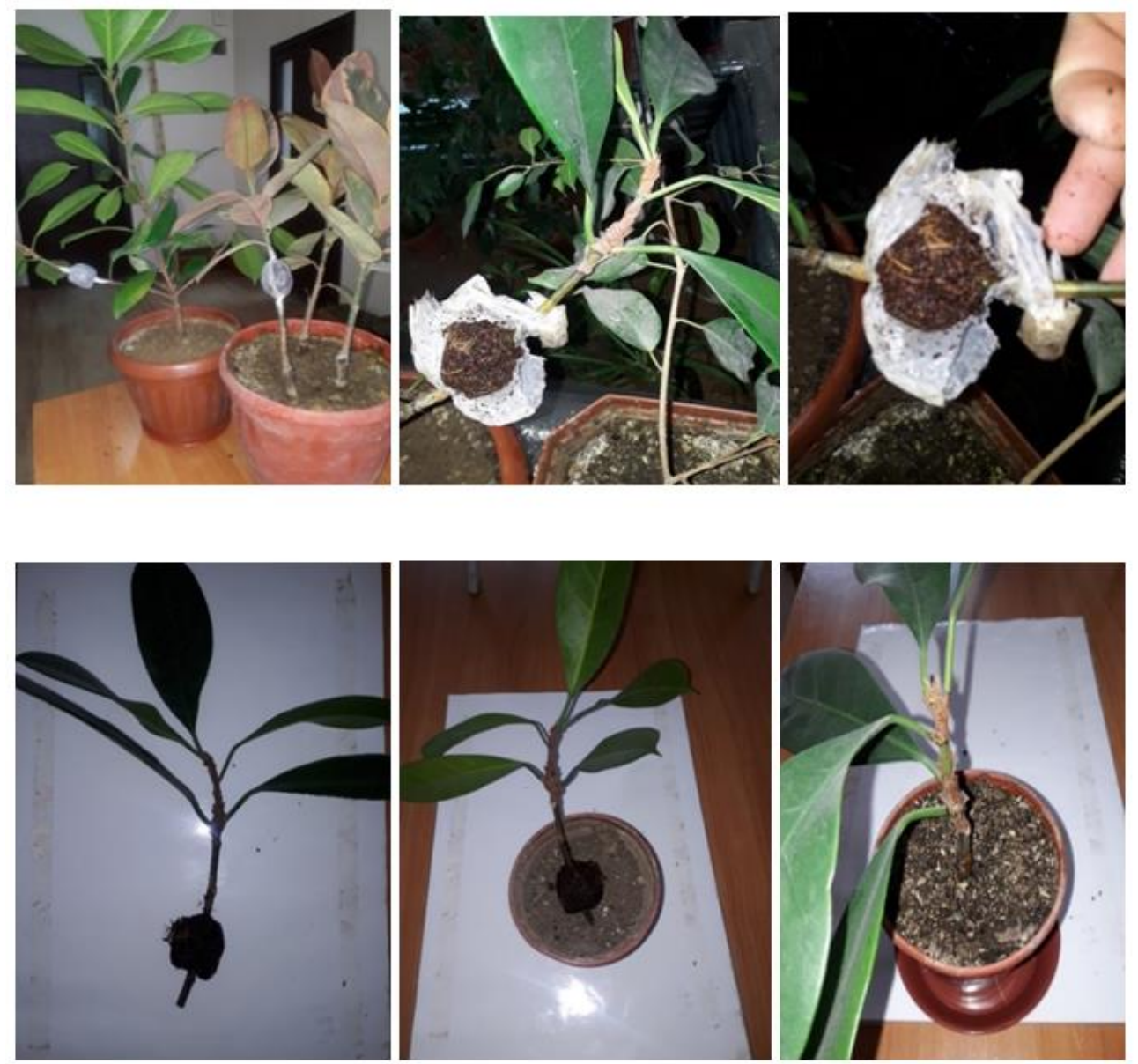

Fig. 3. Propagation of elastic ficus (Ficus elastica) using a suspension link.

Among these measures, soil and sand moisture are of particular importance in plant growth. When moistening the soil and sand, the moisture content was maintained in the order of $45-55 \%, 55-65 \%, 65-75 \%$ relative to the boundary moisture capacity of the soil, while wetting was carried out by spraying (water efficiency $=0.7$ according to A.N Kostyakov [4]). 


\section{Conclusions}

All species of ficus are leaf ornamental species. They rarely produce seeds in indoor conditions. Therefore, they can be propagated vegetatively. Based on the results of experiments, when propagating ficus species, it is necessary to ensure that the room temperature is $18-22^{\circ} \mathrm{C}$, the relative humidity of the room air is $40-50 \%$, the cuttings take root and new leaves emerge. For propagation in sand and soil and other similar compounds, it is recommended to use wooden containers or special plastic containers with a height of $15-20 \mathrm{~cm}$, size $0.5 \times 1.0,1 \times 1,1 \times 2$ meters. All options except for cuttings placed in the soil in the propagation of ficus benjamina plant gave results in the range of $70-90 \%$. Of these, IBA, which has the highest performance, is recommended for multiplication in stimulant water. For growing indoor plants is recommended substrate in the ratio of $1: 1: 1: 1$ of soil, peat, coconut bark, and hydrogel. To avoid damage to the roots of the plant, it is recommended that the water temperature used in irrigation should be $18-22{ }^{\circ} \mathrm{C}$ depending on the room temperature. When propagating ficus species using a suspension bond, it is recommended to use a peat bond. A $1 \mathrm{~cm}$ section of the plant twig is cut with $40 \%$ bark left and a tie is set. It is recommended to check the humidity of the bundle every 2-3 days depending on the room temperature.

\section{References}

1. M.A. Abdou, M.A.H. Mohamed, F.A. Attia, Journal of Agricultural Science, 29 (2),775-785 (2004)

2. A. Abouzari, S. Rouhi, A. Eslami, B. Kaviani, International Journal of Agriculture and Biology, 14(6), 7 (2011)

3. H. Babaie, H. Zarei, K. Hemmati, Journal of Ornamental Plants, 4(2), 75-79 (2014)

4. E. Berdiyev, Agroprocessing, 4(1), 11 (2019)

5. S. Ercisli, O. Anapali, A. Esitken, U. Sahin, Gartenbauwissenschaft, 67(1), 34-38 (2012)

6. M. Urmanova, A. Kuziev, D. Burkhanova, D. Kadirova, N. Namozov, N. Shadieva, E3S Web of Conferences, 244, 02036 (2021)

7. S. Islamov, N. Namozov, M. Saidova, D. Kodirova, E3S Web of Conferences, 244, 03028 (2021)

8. E. Gabryszewska, R. M. Rudnicki, III International Symposium on Artificial Lighting in Horticulture, 418, 163-168 (1994)

9. H.T. Hartman, D. Kester, F.Jr. Davies, R.L. Geneve, Plant propagation: Principles and Practices, 647 (2002)

10. T. Kayumov, S. Egamberdiyev, Agroprocessing, 4(1), 7 (2019)

11. N. Namozov, M. Tursinbaev, I. Yuldoshev, S. Yuldasheva, E3S Web of Conferences, 244, 02007 (2021)

12. E. Mbosso, J. Nguedia, F. Meyer, B. Lenta, S. Ngouela, B. Lallemand, R. Wintjens, Phytochemistry, 83, 95-103 (2012)

13. M. Karimov, N. Namozov, B. Teshaboev, E3S Web of Conferences, 244, 02025 (2021)

14. E. Veneklass, M. Santos Silva, F. Den Ouden, Scientia Horticulturae, 93, 75-84 (2002) 